Chirurg 2013 $\cdot 84: 800$

DOI 10.1007/s00104-013-2584-y

Online publiziert: 11. August 2013

c) Springer-Verlag Berlin Heidelberg 2013

O. Strobel · M.W. Büchler

Klinik für Allgemein-, Viszeral- und Transplantationschirurgie, Universität Heidelberg

\title{
Fast-Track verbessert die Ergebnisse nach Leberresektionen
}

\section{Ergebnisse einer randomisiert kontrollierten Studie}

- eine präoperative kohlenhydratreiche Ernährung und ein früher postoperativer Kostaufbau,

Jones C, Kelliher L, Dickinson M et al (2013) Randomized clinical trial on enhanced recovery versus standard care following open liver resection. Br J Surg 100:1015-1024

\section{Hintergrund und Fragestellung}

In der kolorektalen Chirurgie führen FastTrack-Programme durch optimierte perioperative Behandlung zu einer beschleunigten Rekonvaleszenz und insgesamt verbesserten Ergebnissen. Im Märzheft hatten wir eine beobachtende Studie kommentiert, die an 100 Patienten die Durchführbarkeit und Sicherheit eines Fast-TrackProgramms bei Leberresektionen untersuchte [1]. Der Einfluss des Fast-Track auf die postoperativen Ergebnisse blieb jedoch unklar. Jones et al. führten nun die erste randomisiert kontrollierte Studie zum Vergleich der Ergebnisse eines FastTrack-Programms vs. perioperativer Standardtherapie bei Leberresektionen durch.

\section{Methoden}

Zwischen 03/2011 und 05/2012 wurden 105 Patienten gescreent. Patienten mit offenen Leberresektionen wurden in die Gruppen „Fast-Track“ ( $\mathrm{n}=46)$ und "Standard“ $(n=45)$ randomisiert. Patienten mit inoperablem Befund, mit laparoskopischer Resektion und mit mehrzeitigen Eingriffen wurden ausgeschlossen. Alle Patienten erhielten eine standardisierte Anästhesie und einen Periduralkatheter (PDK). Wichtige Bestandteile des Fast-Track-Programms waren:

- eine ausführlich präoperative Aufklärung der Patienten insbesondere über Kostaufbau und Mobilisation,
- eine zielorientierte postoperative Flüssigkeitssubstitution,

- frühe Mobilisation und Physiotherapie sowie

- Entfernung von PDK und Drainagen am 2. postoperativen Tag.

Primäre Endpunkte waren die Zeit bis zur medizinischen Entlassfähigkeit und die postoperative Krankenhausverweildauer. Als sekundäre Endpunkte wurden unter anderem Morbidität und Mortalität, Schmerz und Lebensqualität untersucht.

\section{Ergebnisse}

Trotz der Randomisierung hatten Patienten in der Fast-Track-Gruppe häufiger größere Resektionen (21 Resektionen >3 Segmente in der Fast-Track-Gruppe vs. 12 in der Standard-Gruppe), häufiger kolorektale Lebermetastasen (35 vs. 26) und häufiger eine vorangegangene Chemotherapie (36 vs. 25). Trotzdem waren die Ergebnisse in der Fast-Track-Gruppe besser: Sowohl die mediane Dauer bis zur medizinischen Entlassfähigkeit (Fast-Track: 3 Tage vs. Standard: 6 Tage) als auch die mediane postoperative Krankenhausverweildauer (FastTrack: 4 Tage vs. Standard: 7 Tage) waren kürzer. Die Rate an nichtchirurgischen Komplikationen war in der Fast-TrackGruppe signifikant reduziert (Fast-Track: $7 \%$ vs. Standard: $27 \%$ ) bei vergleichbarer chirurgischen Komplikationsrate (15\% vs. $11 \%)$ und vergleichbarer Mortalität $(n=1$ in beiden Gruppen). Auch die Lebensqualität in den ersten 28 Tagen war in der FastTrack-Gruppe signifikant besser. Die Wiederaufnahmerate war in der Fast-TrackGruppe nicht signifikant erhöht (2 vs. 0).

\section{Diskussion und Fazit}

Die Autoren folgern, dass Fast-Track-Prinzipien bei Patienten mit Leberresektionen sicher und effektiv umsetzbar sind und zu einer beschleunigten Erholung, einer schnelleren Entlassung, einer Reduktion der nichtchirurgischen Komplikationen sowie zu einer Verbesserung der kurzfristigen Lebensqualität führen. Offen bleibt, ob diese positiven Effekte auf einzelne Komponenten des angewandten Fast-Track-Programms oder auf das Programm als Ganzes zurückzuführen sind. Sicher ist beispielsweise, dass die kurzen Verweildauern im Fast-TrackProgramm nur durch eine konsequente Compliance mit den Fast-Track-Kriterien, insbesondere durch die frühe Entfernung des PDK und der Drainagen möglich werden.

\section{Korrespondenzadresse}

\section{PD Dr. O. Strobel}

Klinik für Allgemein-, Viszeralund Transplantationschirurgie, Universität Heidelberg, Im Neuenheimer Feld 110, 69120 Heidelberg Oliver.Strobel@med.uni-heidelberg.de

\section{Einhaltung der ethischen Richtlinien}

Interessenkonflikt. O. Strobel und M.W. Büchler geben an, dass kein Interessenkonflikt besteht.

Dieser Beitrag beinhaltet keine Studien an Menschen oder Tieren.

\section{Literatur}

\footnotetext{
1. Schultz NA, Larsen PN, Klarskov B et al (2013) Evaluation of a fast-track programme for patients undergoing liver resection. Br J Surg 100:138-143
} 